\title{
Multi-scale modelling of polymer-based optoelectronic devices
}

\author{
Marta M. D. Ramos ${ }^{1 *}$, Helena M. G. Correia ${ }^{1}$, Hélder M. C. Barbosa ${ }^{1}$ \\ ${ }^{1}$ Departamento de Física, Universidade do Minho, Campus de Gualtar, 4710-057 Braga, Portugal
}

\begin{abstract}
The optimization of polymer-based optoelectronic devices such as light-emitting diodes (LEDs), photodetectors and photovoltaic cells requires the understanding how molecular properties and the spatial arrangement of the conjugated strands affect the electronic processes underlying the functioning of these devices. Since some of the important features are determined largely by the individual molecular strands and other features depend strongly on the nanostructure, a multi-scale modelling of materials and device properties is needed. In this work we discuss the atomistic and nanoscale modelling of charge injection, transport and trapping single-carrier diode based on poly(p-phenylene venylene) (PPV), which also applies to other optoelectronic devices.
\end{abstract}

Keywords: Modelling, Semiconductor polymer, Nanostructure, Current efficiency

\section{INTRODUCTION}

Over the past years it became clear the importance of controlling polymer film morphology in electronic and optoelectronic devices such as light-emitting diodes (LEDs) or field-effect transistors (FETs), to improve their performance $[1,2]$. In a pristine conjugated polymer layer without any treatment to control directly the orientation of the polymer chains, the polymer bulk has a "spaghetti-type structure" [3] with an entanglement of chains. Each one of them are folding and bending, which leads to the formation of stiff-chain segments with different conjugation lengths. As a result, the polymer film can be seen as an assemble of domains where the polymer strands can be aligned or have a random orientation to each other, which influences its electric properties. The size of each domain and the orientation of the polymer strands relative to the substrate depends on the experimental conditions used to deposit the polymer on the substrate (e.g. the deposition technique, the solvent, the post-deposition treatment, etc.). To improve the efficiency of devices based on a semiconductor polymer thin film, it has been demonstrated that by controlling the orientation of the conjugated polymer segments, by mechanical alignment or the use of specific substrates, it is possible to improve charge-carrier mobility [4] or to obtain polarized electroluminescence displays [5]. Although at the experimental level it seems clear the benefits of polymer strand alignment, there is not a clear picture of how the orientation of the aligned polymer strands relative to the electrodes at nano scale affects the electric properties of the polymer-based devices. By using a generalized dynamical Monte Carlo method, that takes into account the nanostructure and molecular properties of the polymer, we study the influence of the orientation of the conjugated polymer segments on the electric behaviour of single-carrier diodes based on poly(p-phenylene venylene) (PPV), which also applies to other optoelectronic devices such as polymer LEDs.

\section{DEVICE MODEL AND SIMULATION METHOD}

The usual way to deposit the active component used in polymer diodes is by spin-coating. Although this technique leads to a preferential orientation of the conjugated polymer segments parallel to the electrodes surface (parallel morphology) [6], it has been demonstrated that is possible to decrease the fraction of non-oriented segments (random morphology) within the polymer film by applying a mechanical force [7]. To study the influence of polymer strand alignment on the electric properties of a single-carrier diode we built 3D networks of PPV stiff-chain segments placing them randomly in the gap between two planar electrodes, separated by $100 \mathrm{~nm}$ (model axis), with a specific orientation of the molecular axis relative to the electrodes surface. In order to mimic the parallel morphology, the molecular axis of all polymer strands is constrained to be parallel to the electrodes surface, whereas a random orientation is allowed for the random

\footnotetext{
*marta@fisica.uminho.pt; phone +351253604330; fax +351253678981
} 
morphology. The length of each polymer segment was taken from a Gaussian distribution of lengths with mean value of 7 monomers, being the minimum distance between the molecular axis of two segments of $0.650 \mathrm{~nm}$ [8].

In our model we consider that both charge injection/collection from/by the electrodes and charge transport between polymer strands is mediated by a hopping process with a frequency given by [8]:

$$
w_{i j}=w_{0} \times h(\theta) \times g\left(r_{i j}\right) \times f\left(\Delta \varepsilon_{i j}\right)
$$

where $w_{0}$ is a pre-factor that represents the attempt-to-escape frequency, $h(\theta)$ represents the influence of the angle between the direction of the local electric ( i.e. the sum of the applied electric field, the field due to charge distribution within the polymer network and the field due to electrodes polarization field) and the hop direction, $g\left(r_{i j}\right)$ represents the influence of the hop distance and $f\left(\Delta \varepsilon_{i j}\right)$ represents the influence of the energy barrier height for hopping, which depends on the ionization potential/electron affinity of the polymer strands involved on the hopping of holes/electrons and takes into account the bias voltage between the hopping sites. Only the process with the highest hopping frequency takes place. When a charge is injected into a polymer strand it moves towards its centre unless the local electric field is higher than the field needed to move it towards the strand end, occurring intramolecular charge transport. Ohmic contacts at both electrode/polymer interfaces are considered in this work. More details about the injection/collection and transport processes and the molecular calculations of the properties used as input parameters in this work can be found in Ref. $[3,8,9]$.

To simulate the time evolution of the injected charge (electrons or holes), we attribute to each hopping event a waiting time given by:

$$
\tau=-\frac{\ln (X)}{w_{i j}}
$$

being $X$ a random number uniformly distributed between 0 and 1 , based on based on the first reaction method (FRM) [10].

On the first computer iteration a charge is injected from the electrode and the time is set to zero. The following hopping events have a time of occurrence given by equation 2, and a queue of events with increasing waiting time is established. At each computer iteration the event with the smallest time of occurrence takes place and it is removed from the queue. This time of occurrence is then subtracted to the waiting time of the other events and a new event is inserted in the queue. When a charge reaches the electrode opposite of the injection electrode, and the collection event takes place, all the events associated to this charge are removed from the queue. The simulation finish when the steady state is reached (i.e. the charge density $(\rho)$ inside the polymer layer remains roughly constant) and the simulation time allowed is over. To be possible to compare the results obtained for all morphologies and applied electric fields, we have considered in this work a simulation time of $0.1 \mu \mathrm{s}$.

\section{RESULTS AND DISCUSSION}

To understand the influence of polymer strand orientation relative to the electrodes surface in single-carrier (electrons or holes) polymer diodes, we performed computer experiments of charge injection and transport in PPV diodes using the mesoscopic model described above, for an applied electric field (bias voltage) ranging from $0.35 \mathrm{MV} / \mathrm{cm}(3.5 \mathrm{~V})$ to 0.75 $\mathrm{MV} / \mathrm{cm}(7.5 \mathrm{~V})$. Since the results obtained for electrons and holes are similar, we will focus our discussion on the results obtained for holes. 


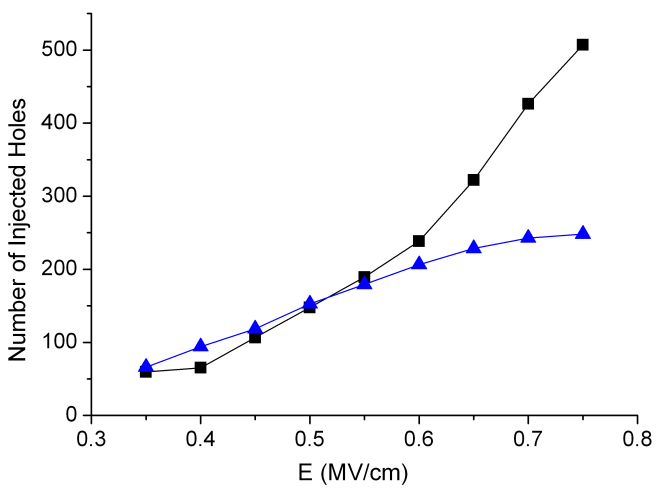

Figure 1. Total number of holes injected into the PPV layers with parallel (square) and random (triangle) morphologies, as a function of the external applied electric field. The lines are just a guide to the eyes.

Figure 1 shows the number of holes injected for the parallel and random morphologies as a function of the applied electric field. For both morphologies, an increase of the applied electric field leads to an increase on the number of holes injected in the polymer layer. Although for applied electric fields smaller than $0.5 \mathrm{MV} / \mathrm{cm}$ the number of injected holes is not significantly different in both polymer morphologies, for higher applied electric fields the rate of hole injection in the polymer layer with parallel morphology is higher than for random morphology. The reason for this different behaviour for larger applied electric fields is due to the competition between the intramolecular and intermolecular charge transport and the effects of energy barrier height for hole hopping. In the case of parallel morphology, hole injection and transport is mainly controlled by the energy barrier height, because the external electric field has the direction perpendicular to the strand axis, not favouring intramolecular charge transport. Since an increase of the applied electric field will reduce the energy barrier height for intermolecular charge transport along its direction, there is a decrease of the charge percolation distance throughout the network which reduces charge transit time. Although an increase of the applied electric field also reduces the energy barrier height for charge hopping in polymer layers with random morphology, charges will stay longer inside the polymer network, since in this case intramolecular charge transport can occur, which will increase charge transit time. The differences in charge transit time inside the network for both polymer morphologies at high applied electric fields will influence charge density inside the polymer layer and, as a consequence, the effect of space charge distribution on the injection process, which limits the amount of charge injection for the random morphology.

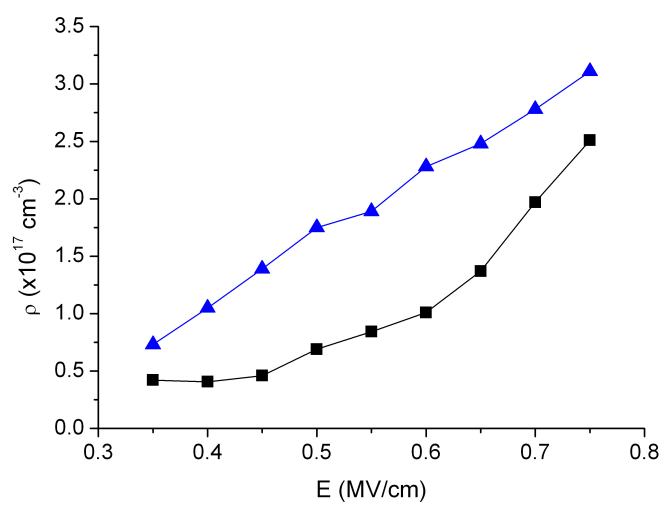

Figure 2. Density of charge at the end of the simulation in the polymer layers with parallel (square) and random (triangle) morphologies, as a function of the external applied electric field. The lines are just a guide to the eyes. 
The differences in charge density for both polymer morphologies are shown in Figure 2. For all the applied electric fields, the charge density for the random morphology is higher than for the parallel morphology, and it increases almost linearly with the strength of the applied electric field, for the random morphology. Since an increase of the applied electric field will increase more the injection rate than the charges transit time in the parallel morphology, this behaviour will lead to a non-linear increase of the charge density with the applied field for such morphology.

The effect of the chain orientation relative to the electrodes is also notorious on both spatial distribution of charge density and the internal electric field (see Fig. 3). Our results show that the polymer layer with the random morphology tends not just to store more charge but also limits its percolation along the network which reduces more the internal electric field near the anode (position at $100 \mathrm{~nm}$ ) as compared to parallel morphology. Similar results where obtained for all the applied electric fields studied in this work.

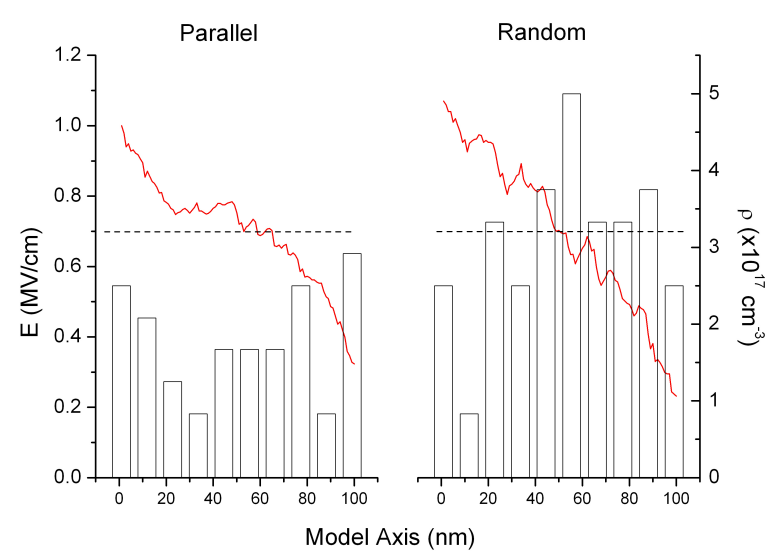

Figure 3. The distribution of charge density along the axis perpendicular to the electrodes surface (model axis) for the parallel and random morphologies. The solid lines represent the internal electric field and the dash lines represent the external applied electric field $(0.7 \mathrm{mV} / \mathrm{cm})$.

\section{CONCLUSIONS}

Although from the experimental point of view it is clear the benefits of aligning polymer chains parallel to the electrodes surface to improve the performance of electronic devices, the reason for such improvement is not well understood. In this work we used a dynamic charge transport model of single-carrier polymer diodes, in which polymer film morphology and the parameter related to molecular properties are explicitly included, to asses the influence of strand orientation relative to electrodes surface on charge injection and charge trapping. Our results show that for a PPV diode the charge injection deviate from linearity at applied electric fields greater that $0.5 \mathrm{MV} / \mathrm{cm}$, for the case of polymer strands with their long axis parallel to the electrodes surface due to: i) an increase of charge transport inside the polymer layer; ii) a decrease of charge density and thus an increase of local electric field near the electrode that improves charge injection. Although these results were obtained with a pristine polymer layer and in the presence of perfect ohmic contacts, our model has proved very helpful to assess the influence of polymer morphology on the electric properties of polymerbased diodes which can not be obtained directly from the experiments.

\section{KNOWLEDGEMEN}

This work is part of the research projects POCTI/CTM/41574/2001 and CONC-REEQ/443/EEI/2005, approved by the Portuguese Foundation for Science and Technology (FCT) and support by the European Community Fund FEDER. One of us (H.M.G.C.) is also indebted to FCT for financial support under the post-doctoral grant no. SFRH/BPD/64554/2009. 


\section{REFERENCES}

[1] Benanti, T. L., and Venkataraman, D., "Organic solar cells: An overview focusing on active layer morphology," Photosynthesis Research, 87(1), 73-81 (2006).

[2] Jaiswal, M., and Menon, R., "Polymer electronic materials: a review of charge transport," Polymer International, 55(12), 1371-1384 (2006).

[3] Stoneham, A. M., Ramos, M. M. D., Almeida, A. M. et al., "Understanding electron flow in conducting polymer films: injection, mobility, recombination and mesostructure," Journal of Physics-Condensed Matter, 14(42), 9877-9898 (2002).

[4] Marsh, R. A., Groves, C., and Greenham, N. C., "A microscopic model for the behavior of nanostructured organic photovoltaic devices,” Journal of Applied Physics, 101(8), 7 (2007).

[5] Grell, M., and Bradley, D. D. C., "Polarized luminescence from oriented molecular materials," Advanced Materials, 11(11), 895-905 (1999).

[6] Ramsdale, C. M., and Greenham, N. C., "Ellipsometric determination of anisotropic optical constants in electroluminescent conjugated polymers," Advanced Materials, 14(3), 212-+ (2002).

[7] Backlund, T. G., Sandberg, H. G. O., Osterbacka, R. et al., "A novel method to orient semiconducting polymer films," Advanced Functional Materials, 15(7), 1095-1099 (2005).

[8] Ramos, M. M. D., and Correia, H. M. G., "Modelling the effects of molecular arrangements in polymer lightemitting diodes," Journal of Physics: Condensed Matter, 18, S429-S438 (2006).

[9] Barbosa, H. M. C., and Ramos, M. M. D., "Mesoscopic modelling of polymer-based optoelectronic devices," Plasma Processes and Polymers, 4, S104-S107 (2007).

[10] Lukkien, J. J., Segers, J. P. L., Hilbers, P. A. J. et al., "Efficient Monte Carlo methods for the simulation of catalytic surface reactions," Physical Review E, 58(2), 2598-2610 (1998). 